\title{
Candida periprosthetic joint infections - risk factors and outcome between albicans and non-albicans strains
}

\author{
Daniel Karczewski ${ }^{1}$-Yi Ren ${ }^{1} \cdot$ Octavian Andronic ${ }^{2} \cdot$ Doruk Akgün$^{1} \cdot$ Carsten Perka ${ }^{1} \cdot$ Michael Müller $^{1}$. \\ Arne Kienzle ${ }^{1,3}$ (D)
}

Received: 11 June 2021 / Accepted: 7 September 2021 / Published online: 16 November 2021

(c) The Author(s) 2021

\begin{abstract}
Background Despite its scarcity, fungal periprosthetic joint infection (PJI) is of great clinical relevance as diagnosis and treatment are highly challenging. Previous analyses focused on the treatment rather than the role of the causative fungal agent on clinical outcome. This is the largest study of its kind to evaluate Candida strain-dependent differences in patients with fungal PJI. Methods We retrospectively analyzed 29 patients who underwent surgical intervention due to Candida hip or knee PJI in our department from 2010 to 2018. PJI was defined according to IDSA, recurrent PJI according to modified Delphi consensus criteria. Statistical analysis was performed using $t$-test, chi-square test with Yates correction, and log rank test.

Results Besides age and affected joint, no significant differences were found between Candida albicans and non-albicans PJI patients ( 75.83 versus 64.11 years, $p=0.012 ; 12$ hip versus two knee cases, $p=0.013$ ). Most patients received two$(27.59 \%)$ or three-stage exchange surgery $(41.38 \%)$. There was a statistical trend towards an increase in surgery needed in non-albicans Candida PJI (2.92 versus 2.12; $p=0.103$ ). After initial Candida PJI treatment, functional prosthesis implantation was achieved in $72.41 \%$ of all patients. At last follow-up, infection-free survival was at $26.79 \%$ in Candida albicans versus $72.00 \%$ in non-albicans PJI $(p=0.046)$.

Conclusions In this study, we found infection-free survival rates to be significantly decreased in patients with albicans compared to non-albicans Candida PJI. While age and affected joint might play a confounding role, we speculate the causative pathogen to play a decisive role in disease progression.
\end{abstract}

Keywords Periprosthetic joint infection · Candida $\cdot$ Fungal infection $\cdot$ Revision arthroplasty $\cdot$ Total knee arthroplasty · Total hip arthroplasty

\section{Abbreviations}

CRP C-reactive protein

PJI Periprosthetic joint infection

TKA Total knee arthroplasty

THA Total hip arthroplasty

DAIR Debridement, antibiotics, and implant retention

Arne Kienzle

arne.kienzle@charite.de

1 Center for Musculoskeletal Surgery, Clinic for Orthopedics, Charité University Hospital, Charitéplatz 1, 10117 Berlin, Germany

2 Department of Orthopaedics, Balgrist University Hospital, University of Zurich, Forchstrasse 340, 8008 Zurich, Switzerland

3 Berlin Institute of Health at Charité - Universitätsmedizin Berlin, BIH Biomedical Innovation Academy, Charitéplatz 1, 10117, Berlin, Germany

\section{Introduction}

Despite increased routine usage of antibiotic prophylaxis and improved aseptic surgical techniques, periprosthetic joint infection (PJI) is still a common yet severe complication after both TKA and THA [1-5]. While the vast majority of cases are made up by bacterial infections, in approximately $1 \%$ of all cases, PJI is due to fungal microorganisms $[6,7]$. In spite of its scarcity, detailed understanding of fungal PJI is of great clinical relevance as diagnosis and treatment is highly challenging for physicians and patients alike [8]. Additionally, treatment of fungal PJI is usually complicated by additional severe diseases impacting the immunocompetence in these affected patients $[9,10]$. While an interdisciplinary team of surgeons, microbiologists, and infectious disease specialists is considered optimal, patients often undergo several procedures until being diagnosed and treated accordingly at a specialized centre $[11,12]$. As there 
are no biofilm-active antimycotics available, surgical intervention and antifungal therapy are obligatory to achieve a successful, infection-free outcome [13, 14]. Revision surgery typically comprises removal of the infected prosthesis, debridement, irrigation, and introduction of a temporary arthrodesis followed by reimplantation of a new prosthesis in a second-stage surgery [15]. While there is broad consensus regarding treatment of bacterial PJI, there is paucity of data to validate an agreed upon treatment protocol for fungal PJI [16]. Besides prosthesis exchange surgery, single case reports have suggested implant retention or permanent resection arthroplasty of the hip and arthrodesis of the knee joint to be viable treatment options [17-22].

A range of case reports and smaller case series have investigated patient demographics and outcomes in patients suffering from fungal PJI [20, 23, 24]. However, previous systematic analyses have focused on the impact of treatment rather than the role of the causative fungal agent on clinical outcome $[9,10]$. Fungal PJI comprises a range of different microorganisms. Commonly, fungal PJI is due to various Aspergillus or Candida strains [25]. While in most cases fungal PJI is caused by Candida [6, 18], relative prevalence of the different Candida strains in PJI is unknown. Besides Candida albicans, cases of Candida freyschussii, Candida glabrata, Candida tropicalis, and Candida parapsilosis PJI have been previously described [26-30]. However, despite its clinical relevance, little research has focused on systematically exploring differences in distribution, clinical features, and outcome. This lack of knowledge on Candida strain-dependent patient characteristics and clinical outcome leads to a paucity in differential and targeted treatment approaches.

In this study, we retrospectively analyzed the cases of 29 patients who underwent surgical intervention due to Candida PJI. This is the largest study of its kind to evaluate Candida strain-dependent differences in patients affected by this pathology. Patient demographics, risk factors, clinical and paraclinical parameters, and long-term outcome were assessed and compared for patients suffering from Candida albicans PJI and non-albicans Candida PJI.

\section{Material and methods}

\section{Patients}

This study was approved by the institutional ethics board and completed in accordance with the Declaration of Helsinki. We retrospectively studied all patients that were diagnosed with PJI with a Candida strain and subsequently underwent PJI-dependent TKA or THA revision surgery in between 2010 and 2018 at our hospital. A total of 29 patients were included in this study. Exclusion criterion was the following: diagnosed PJI lacking positive microbiological culture for any Candida strain. There were no further exclusions. Clinical and paraclinical parameters were evaluated for all patients. PJI was defined according to IDSA criteria as previously described [31]. For all statistical assessments in this study, patients were divided into the two groups depending on the detected Candida strain: Candida albicans PJI and non-albicans Candida PJI patients.

\section{Treatment}

Patients received centralized and interdisciplinary treatment in our specialized department for total joint replacement and infections. Antibacterial and antifungal therapies were initiated as soon as PJI was diagnosed and was continuously adapted according to found microbial susceptibilities and resistances. Antimicrobial treatment was chosen based on bacterial susceptibility, the recommendations of Zimmerli [32], and in consultation with our department for microbiology and infectiology.

For revision surgery, one-, two-, or multiple-stage exchange surgery was performed as previously described $[15,33,34]$. After removal of the infected prosthesis, patients received a temporary, antibiotic-loaded cement spacer between stages in case of two- or multiple-stage exchange surgery. Antibiotics were chosen based on microbial susceptibilities and resistances or empirically. Prosthesis reimplantation was performed at least six weeks after removal with no clinical and laboratory signs of infection apparent. In few cases, permanent girdlestone resection arthroplasty or DAIR was performed at patients' behest or if reimplantation was not possible due to paraclinical parameters. All surgical procedures were conducted by high-volume surgeons specialized in the treatment of PJI. Postoperatively, all patients received long-term antifungal suppression treatment through our outpatient department or were advised to continue treatment through their local orthopaedic doctor.

\section{Microbiology}

Blood samples, joint aspirate, and intra-operative specimens were assessed by our clinical medicine and microbiology department. Patients were grouped depending on the detected fungal pathogen into Candida albicans and non-albicans Candida PJI patients. Pathogens found in latter group consisted of Candida tropicalis, Candida parapsilosis, and Candida guilliermondii. Mixed fungal infections with both Candida albicans and non-albicans Candida were diagnosed in two cases and were assigned to the Candida albicans group. 


\section{Follow-up}

All patients were regularly invited to our specialized outpatient clinic for follow-up examinations. Patients were invited six to 12 weeks post-operatively and thereafter for annual follow-ups. Patients that could not visit our outpatient department due to severe comorbidities were invited to follow-ups by phone. Complications of revision arthroplasty comprise, among others, recurrent PJI with the same or a different pathogen, aseptic loosening, instability, pain, patellar maltracking, arthrofibrosis, and limited range of motion. For this study, endpoints were defined as recurrent PJI, revision surgery-, or PJI-associated death. Recurrent PJI was diagnosed using IDSA and modified Delphi criteria [31, 35].

\section{Statistics}

All data were analyzed using GraphPad Prism 7 software (GraphPad Software, La Jolla, CA, USA) and Excel (v16.30; Microsoft Corporation, Redmond, WS, USA). All data are presented as mean \pm one standard deviation. Statistical analysis was performed using one-way analysis of variance. Unpaired Student's $t$-test for samples of unequal variances, and chisquared test with Yates correction was used to test for statistical significance $(p<0.05)$. Where applicable, $p$ values are listed.

\section{Results}

\section{Patient characteristics}

Patient characteristics are outlined in Table 1. Of the 29 patients included in our analysis, 13 were male and 16 were female. Average patient age was $70.98 \pm 12.72$ years. Mean duration of follow-up after the last surgical intervention was 33.03 months (range, 0.37 to 119.13 months). Affected joints were the hip in 14 patients and knee in 15 patients. Mean Charlson Comorbidity Index (CCI) was 5.38 (range,
2 to 10). Most patients had an ASA score of $2(13.79 \%)$ or $3(82.76 \%)$.

For all statistical analyses, patients were grouped into patients suffering from Candida albicans PJI versus nonalbicans Candida PJI. Patients in the Candida albicans group were significantly older $(+11.72$ years; $p=0.012)$ compared to the non-albicans Candida patients. Candida albicans PJI affected the hip joint significantly more often, while in the non-albicans Candida group, the knee joint was most affected. No significant differences for any of the other analyzed parameters were found.

\section{Diagnosis and treatment of PJI}

Pathologically elevated CRP ( $>5 \mathrm{mg} / \mathrm{L})$ was found in all but three patients pre-operatively (Table 2). Mean CRP was $51.74 \mathrm{mg} / \mathrm{L}$ (range, 3.70 to $249.40 \mathrm{mg} / \mathrm{L}$ ). Pre-operative prevalence of leukocytes was at physiological levels (normal range 3.90 to 10.50 cells $/ \mathrm{nL}$ ) in all but four patients that showed leukocytosis. No significant differences in CRP (range, 4 to $249 \mathrm{mg} / \mathrm{L}$ ) and leukocytes (range, 11.10 to 26.10 cells $/ \mathrm{nL}$ ) were found in between Candida albicans PJI and non-albicans Candida PJI patients ( $p=0.111$ and $p=0.133$ ). Polymicrobial infection with additional bacterial pathogens was found in $75.86 \%$ of all cases $(76.47 \%$ for Candida albicans PJI and $75.00 \%$ for non-albicans Candida PJI).

A substantial number of patients had received revision arthroplasty due to aseptic loosening (16 patients), non-fungal PJI (14 patients), or - at independent time points - both (1 patient) prior to suffering from Candida PJI (Table 3). In the majority of patients (12 in the Candida albicans group and nine in the non-albicans Candida group), bacterial PJI was diagnosed prior to the Candida PJI diagnosis (mean, 258.10 days; range, 3 to 1273 days). No significant differences in mean time between diagnosis of bacterial and Candida PJI was found for the Candida albicans (mean, 204.75 days) and non-albicans Candida group (mean, 329.22; $p=0.183$ ). In five cases, Candida PJI was the

Table 1 Patient characteristics

\begin{tabular}{|c|c|c|c|c|}
\hline Descriptive & All patients & Candia albicans PJI & Non-albicans Candida PJI & $p$ value \\
\hline Count [number] & 29 & 17 & 12 & - \\
\hline Gender distribution [count] & 13 males; 16 females & 5 males; 12 females & 8 males; 4 females & 0.108 \\
\hline Age [years] & $70.98 \pm 12.72$ & $75.83 \pm 7.29$ & $64.11 \pm 15.71$ & 0.012 \\
\hline Follow-up time [months] & $33.03 \pm 32.80$ & $26.70 \pm 33.48$ & $42.01 \pm 30.95$ & 0.223 \\
\hline Affected joint [count] & Hip: 14; knee: 15 & Hip: 12 ; knee: 5 & Hip: 2; knee: 10 & 0.013 \\
\hline \multicolumn{5}{|l|}{ Clinical scores } \\
\hline CCI & $5.38 \pm 2.09$ & $5.88 \pm 2.00$ & $4.67 \pm 2.10$ & 0.126 \\
\hline ASA1 [\%] (number of patients [count]) & $3.45 \%(1)$ & $0.00 \%(0)$ & $8.33 \%(1)$ & - \\
\hline ASA2 [\%] (number of patients [count]) & $13.79 \%(4)$ & $5.88 \%(1)$ & $25.00 \%(3)$ & - \\
\hline ASA3 [\%] (number of patients [count]) & $82.76 \%(24)$ & $94.12 \%(16)$ & $66.67 \%(8)$ & - \\
\hline
\end{tabular}


Table 2 Laboratory analysis of bloodwork and microbiology

\begin{tabular}{lllll}
\hline Descriptive & All patients & Candia albicans PJI & $\begin{array}{l}\text { Non-albicans } \\
\text { Candida } \text { PJI }\end{array}$ & $p$ value \\
\hline $\begin{array}{l}\text { Laboratory analysis } \\
\text { CRP [mg/L] }\end{array}$ & & & & \\
Leukocytes [cells/nL] & $51.74 \pm 60.57$ & $66.85 \pm 72.53$ & $30.33 \pm 28.88$ & 0.111 \\
$\begin{array}{l}\text { Microbiology } \\
\text { Candida } \text { strain [\%] (Number of Patients [count]) }\end{array}$ & & $7.32 \pm 1.88$ & 0.133 \\
$\quad \sim$ albicans & $58.62 \%(17)$ & $100.00 \%(17)$ & $0.00 \%(0)$ & - \\
$\quad \sim$ glabrata & $3.45 \%(1)$ & $5.88 \%(1)$ & $0.00 \%(0)$ & - \\
$\quad \sim$ parapsilosis & $34.48 \%(10)$ & $0.00 \%(0)$ & $83.33 \%(10)$ & - \\
$\sim$ guilliermondii & & & \\
$\quad \sim$ tropicalis & $3.45 \%(1)$ & $0.00 \%(0)$ & $8.33 \%(1)$ & - \\
$\begin{array}{l}\text { Additional bacterial infection [\%] } \\
\text { (Number of Patients [count]) }\end{array}$ & $75.86 \%(22)$ & $76.47 \%(13)$ & $75.00 \%(9)$ & - \\
\hline
\end{tabular}

Table 3 Previous surgeries and PJI treatment

\begin{tabular}{|c|c|c|c|c|}
\hline Descriptive & All Patients & Candia albicans PJI & $\begin{array}{l}\text { non-albicans } \\
\text { Candida PJI }\end{array}$ & $p$ value \\
\hline \multicolumn{5}{|l|}{ Prior Prosthesis Exchange Surgeries } \\
\hline ...due to non-Candida PJI [\%] (Number of Patients [count]) & $48.28 \%(14)$ & $52.94 \%(9)$ & $41.67 \%(5)$ & - \\
\hline ...due to Aseptic Loosening [\%] (Number of Patients [count]) & $55.17 \%(16)$ & $64.71 \%(11)$ & $41.67 \%(5)$ & - \\
\hline \multicolumn{5}{|l|}{ Surgical treatment } \\
\hline Number of surgical procedures [count] & $2.45 \pm 1.30$ & $2.12 \pm 1.32$ & $2.92 \pm 1.16$ & 0.103 \\
\hline Duration of prosthesis-free Interval [months] & $5.75 \pm 5.81$ & $5.33 \pm 6.82$ & $6.12 \pm 5.09$ & 0.776 \\
\hline Negative pressure wound therapy [\%] (number of patients [count]) & $37.93 \%(11)$ & $41.18 \%(7)$ & $33.33 \%(4)$ & - \\
\hline \multicolumn{5}{|l|}{ Antifungal treatment } \\
\hline ...with fluconazole & $86.21 \%(25)$ & $82.35 \%(14)$ & $91.67 \%(11)$ & - \\
\hline ...with caspofungin & $13.79 \%(4)$ & $17.65 \%(3)$ & $8.33 \%(1)$ & - \\
\hline ...with voriconazole & $6.90 \%(2)$ & $11.76 \%(2)$ & $0.00 \%(0)$ & - \\
\hline Additional antibiotics treatment [\%] (number of patients [count]) & $96.55 \%(28)$ & $94.12 \%(16)$ & $100.00 \%(12)$ & - \\
\hline
\end{tabular}

first diagnosed PJI and, in three cases, time between bacterial and Candida PJI was unknown.

In average, patients received 2.45 surgical procedures and negative pressure wound therapy was administered in $37.93 \%$ of all cases. While the majority of patients received two- $(27.59 \%$ of all patients) or three-stage exchange surgery $(41.38 \%$ of all patients), two patients $(6.90 \%$ of all patients) underwent one-stage exchange surgery or DAIR, respectively. In $17.24 \%$ of all cases, patients did not receive a new prosthesis after implant removal. There was a statistical trend towards an increase in surgery needed in patients with non-albicans Candida PJI (2.92 versus 2.12 in Candida albicans PJI; $p=0.103$ ).

In case of two- or multiple-stage exchange surgery, mean duration of prosthesis-free interval was 5.75 months (range, 1.53 to 23.13 months) with no significant differences in between groups $(p=0.777)$. Figure 1 shows images of a representative patient who experienced Candida parapsilosis
PJI after primary TKA and received two-stage exchange surgery.

The majority of patients $(86.20 \%)$ received fluconazole alone or in combination with an additional antifungal agent. Caspofungin and voriconazole as sole antifungal therapy were administered to one patient each. At the patients' behest, one patient did not receive antifungal or antibiotic treatment but palliative care instead. All other patients received antibiotic treatment. A minimum of 12 months of antifungal treatment was recommended to all patients at discharge. Individual microbiological results and treatment regimens are detailed in Supplemental Table 1.

\section{Outcome}

After initial Candida PJI treatment, functional prosthesis implantation was achieved in $72.41 \%$ of all patients (Table 4). In eight patients, girdlestone resection 
Fig. 1 Representative patient who experienced Candida parapsilosis $\mathrm{PJI}$ after primary TKA and received two-stage exchange surgery
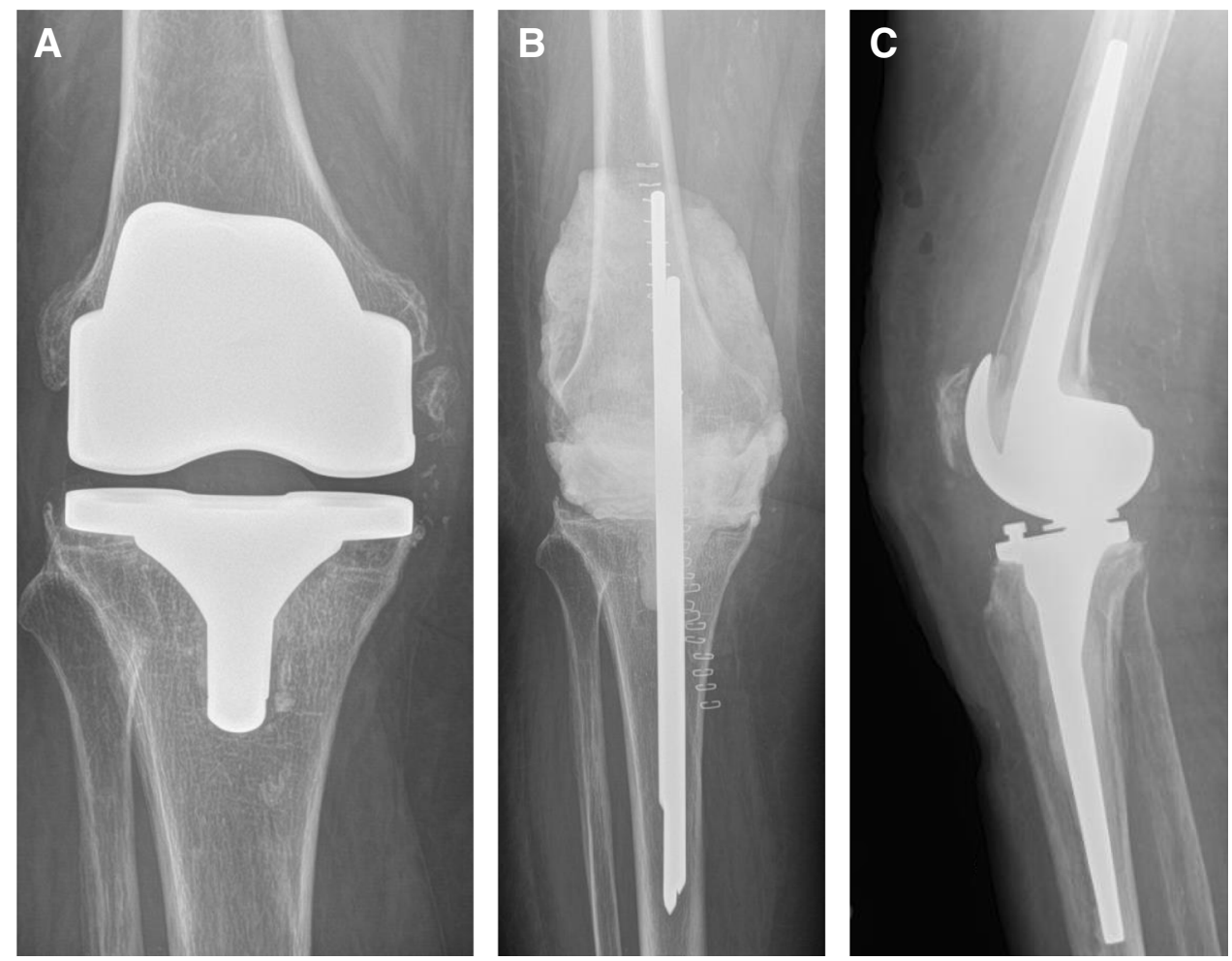

arthroplasty of the hip or arthrodesis of the knee was the final outcome. PJI-associated death was reported for three patients at the last follow-up. No significant differences were found between groups at both time points.

Infection-free survival was significantly lower in patients with Candida albicans PJI (Fig. 2; 26.79\%) compared to patients with non-albicans Candida PJI (72.00\%; $p=0.046)$. Recurrent PJI occurred in a total of eight patients after an average time of 10.37 months (range, 0.17 to 32.63 months): Recurrent PJI occurred in $35.29 \%$ of the patients in the Candida albicans and in $16.67 \%$ of the patients in the non-albicans Candida PJI group (Table 4).

\section{Discussion}

In this study, we assessed clinical and paraclinical data to study potential differences in patient demographics, treatment, and clinical outcome in patients with PJI due to Candida albicans versus non-albicans Candida strains at our university hospital. Besides age and the affected joint, no significant differences were found in patient characteristics and treatment. However, long-term infection-free survival rate was significantly lower in patients with Candida albicans PJI compared to non-albicans Candida PJI.

Table 4 Outcome

\begin{tabular}{|c|c|c|c|c|}
\hline Descriptive & All patients & Candia albicans PJI & $\begin{array}{l}\text { Non-albicans } \\
\text { Candida PJI }\end{array}$ & $p$ value \\
\hline \multicolumn{5}{|l|}{ Outcome after Candida PJI treatment } \\
\hline Prosthesis (THA/TKA) [\%] (number of patients [count]) & $72.41 \%(21)$ & $70.59 \%(12)$ & $75.00 \%(9)$ & 0.793 \\
\hline $\begin{array}{l}\text { Girdlestone resection arthroplasty/arthrodesis [\%] (number of } \\
\text { patients [count]) }\end{array}$ & $27.59 \%(8)$ & $29.41 \%(5)$ & $25.00 \%(3)$ & \\
\hline \multicolumn{5}{|l|}{ Outcome at final follow-up } \\
\hline Prosthesis (THA/TKA) [\%] (number of patients [count]) & $62.07 \%(18)$ & $52.94 \%(9)$ & $75.00 \%(9)$ & 0.367 \\
\hline $\begin{array}{l}\text { Girdlestone resection arthroplasty/arthrodesis/amputation [\%] } \\
\text { (number of patients [count]) }\end{array}$ & $27.59 \%(8)$ & $29.42 \%(5)$ & $25.00 \%(3)$ & \\
\hline PJI-associated death [\%] (number of patients [count]) & $10.34 \%(3)$ & $17.65 \%(3)$ & $0.00 \%(0)$ & \\
\hline Recurrent PJI [\%] (number of patients [count]) & $27.59 \%(8)$ & $35.29 \%(6)$ & $16.67 \%(2)$ & - \\
\hline Time to recurrent PJI (months) & $10.37 \pm 13.02$ & $10.72 \pm 14.98$ & $9.32 \pm 7.94$ & 0.875 \\
\hline
\end{tabular}




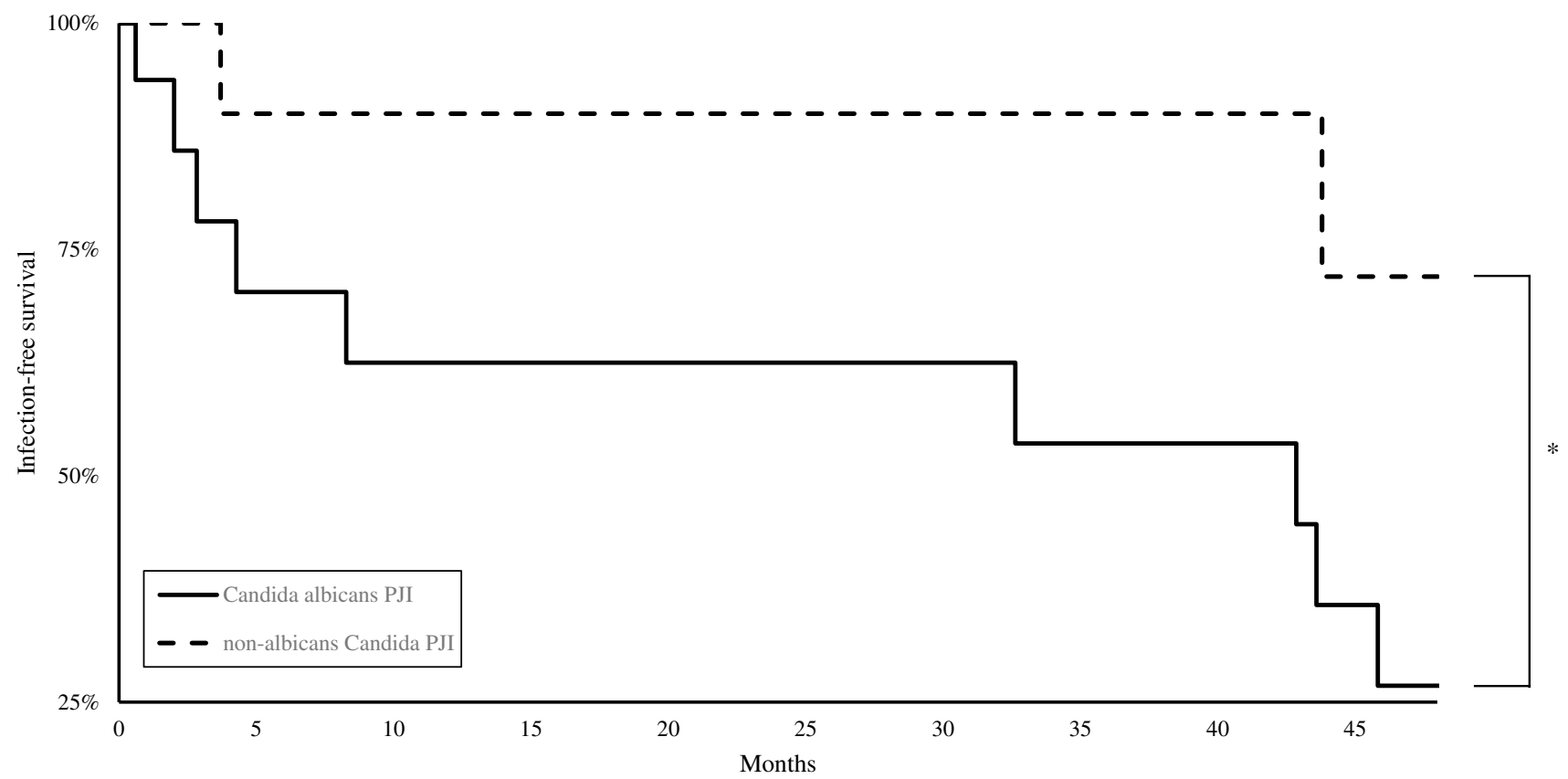

Fig. 2 Candida albicans PJI and non-albicans Candida PJI

In this study, we found Candida albicans followed by Candida parapsilosis to be the most commonly diagnosed Candida strains. While mean age was comparable to patients receiving primary TKA or THA [36], both ASA and CCI scores were higher in our patient collective suggesting comorbidities to be a significant risk factor. However, patients affected by Candida albicans PJI were significantly older compared to non-albicans Candida PJI patients. Of note, we found a substantial number of patients suffering from Candida PJI to have had prior revision arthroplasty due to aseptic loosening or non-fungal PJI. In accordance, with these results, previous reports highlighted an elevated risk for fungal PJI in older patients that had previously received revision arthroplasty [6]. Polymicrobial infections were found in approximately $75 \%$ of the cases in our study, compared to 19 to $36 \%$ in bacterial PJI [37, 38], suggesting impaired immune response in these patients.

While there is a strong tendency towards two- or multiplestage revision surgery, reports on comparable outcomes after single-stage exchange surgery exist [21, 22]. However, due to its scarcity, there is no generally agreed upon treatment guidelines for fungal PJI. As this is the largest study of its kind to evaluate Candida PJI, previous reports have struggled to draw valid conclusions regarding differences in clinical features and outcome from smaller case series [16]. In most cases, treatment is further complicated by impacted immunocompetence in these affected patients $[9,10]$. As evident in our patient collective, Candida PJI is in most cases only diagnosed after initial treatment for bacterial PJI leading to stark differences in surgical treatment. As there are no biofilm-active antimycotics available, re-revision and potentially an exchange to an antimycotic cement spacer might be necessary in such cases [13, 14]. However, if fungal PJI is diagnosed after prosthesis reimplantation, surgeons may opt to attempt to retain the implant with long-term antifungal therapy in severely diseased patients with a high intra-operative risk. With current diagnostic tools and guidelines, it may not be feasible to strictly follow an agreed-upon therapeutic approach in affected patients. This also limits the peri-operative use of antifungal medications and as an additive during cementation. In our experience, involved surgeons should consider fungal pathogens particularly if PJI persists despite adequate antibiotic and surgical treatment. Additionally, as seen in our patients, it may not be possible for severely diseased patients to receive a new implant after resection arthroplasty or arthrodesis due to softtissue constraints. Similarly, previous case reports have suggested permanent resection arthroplasty of the hip and arthrodesis of the knee joint as viable treatment options [17-20]. The lack of an agreed upon treatment protocol for fungal PJI and the resulting large differences in treatment in the literature and in our patient sample further complicates comparison of outcome [16]. Additionally, treatment regimens are commonly chosen depending on the surgeons' experience and sometimes limited at the patients' behest in these often highly complex cases. The lack of larger patient sample size warrants further research on optimal treatment of these patients.

While infection-free survival rates are at approximately 80 to $90 \%$, risk of re-revision due to recurrent PJI or aseptic loosening is significantly elevated after primary bacterial PJI [33, 39]. Reviews on a number of published case reports and smaller case 
series have concluded treatment of fungal PJI to be markedly less successful with infection-free survival rates ranging from approximately 40 to $80 \%[7,16,17]$. In our study, we found similar overall survival rates. However, clinical outcome was at only $27 \%$ in the Candida albicans PJI group. In contrast, infection-free survival in patients with non-albicans Candida PJI was at $72 \%$. Additionally, we found a significantly greater number of patients with PJI of the hip in this group compared to in non-albicans Candida PJI patients. In accordance with these results, Brown et al. found patients with fungal PJI of the hip - independent of the fungal strain - to have significantly lower infection-free survival rates [6]. Previously, both ASA and CCI have been suggested to be useful in predicting risk of fungal PJI after revision arthroplasty [6, 40]. In contrast to these reports, clinical outcomes in both groups did not correlate to ASA or CCI in our patient collective.

Limitations of the current study include the heterogeneity of the analyzed population, the retrospective study design, and the analyzed cohort size with potential subsequent statistical bias.

In conclusion, we found infection-free survival rates to be significantly decreased in patients with Candida albicans PJI compared to non-albicans Candida PJI. While age and affected joint might play a confounding role, we speculate the causative pathogen to play a decisive role in disease progression.

Supplementary Information The online version contains supplementary material available at https://doi.org/10.1007/s00264-021-05214-y.

Acknowledgements Dr. Arne Kienzle is participant in the BIH-Charité Junior Clinician Scientist Program funded by the Charité - Universitätsmedizin Berlin and the Berlin Institute of Health.

Author contribution Conceptualization, Michael Müller and Arne Kienzle; data curation, Daniel Karczewski, Octavian Andronic and Doruk Akgün; formal analysis, Daniel Karczewski and Arne Kienzle; investigation, Yi Ren and Arne Kienzle; methodology, Arne Kienzle; project administration, Arne Kienzle; supervision, Carsten Perka and Michael Müller; validation, Yi Ren; writing — original draft, Arne Kienzle; writing - review and editing, Doruk Akgün, Carsten Perka, Michael Müller and Arne Kienzle.

Funding Open Access funding enabled and organized by Projekt DEAL.

\section{Declarations}

Conflict of interest The authors declare no competing interests.

Open Access This article is licensed under a Creative Commons Attribution 4.0 International License, which permits use, sharing, adaptation, distribution and reproduction in any medium or format, as long as you give appropriate credit to the original author(s) and the source, provide a link to the Creative Commons licence, and indicate if changes were made. The images or other third party material in this article are included in the article's Creative Commons licence, unless indicated otherwise in a credit line to the material. If material is not included in the article's Creative Commons licence and your intended use is not permitted by statutory regulation or exceeds the permitted use, you will need to obtain permission directly from the copyright holder. To view a copy of this licence, visit http://creativecommons.org/licenses/by/4.0/.

\section{References}

1. Delanois RE, Mistry JB, Gwam CU, Mohamed NS, Choksi US, Mont MA (2017) Current epidemiology of revision total knee arthroplasty in the United States. J Arthroplasty 32:2663-2668. https://doi.org/10.1016/j.arth.2017.03.066

2. Kurtz SM, Lau E, Schmier J, Ong KL, Zhao K, Parvizi J (2008) Infection burden for hip and knee arthroplasty in the United States. J Arthroplasty 23:984-991. https://doi.org/10.1016/j.arth. 2007.10.017

3. He W, Kienzle A, Liu XJ, Muller WEG, Feng QL (2015) In vitro $30 \mathrm{~nm}$ silver nanoparticles promote chondrogenesis of human mesenchymal stem cells. RSC Adv 5:49809-49818. https://doi. org/10.1039/c5ra06386h

4. He W, Liu X, Kienzle A, Muller WE, Feng Q (2016) In vitro uptake of silver nanoparticles and their toxicity in human mesenchymal stem cells derived from bone marrow. J Nanosci Nanotechnol 16:219-228. https://doi.org/10.1166/jnn.2016.10728

5. Kienzle A, Biedermann L, Babeyko E, Kirschbaum S, Duda G, Perka C, Gwinner C (2021) Public interest in knee pain and knee replacement during the SARS-CoV-2 pandemic in Western Europe. J Clin Med 10. https://doi.org/10.3390/jcm10051067

6. Brown TS, Petis SM, Osmon DR, Mabry TM, Berry DJ, Hanssen AD, Abdel MP (2018) Periprosthetic joint infection with fungal pathogens. J Arthroplasty 33:2605-2612. https://doi.org/10. 1016/j.arth.2018.03.003

7. Azzam K, Parvizi J, Jungkind D, Hanssen A, Fehring T, Springer B, Bozic K, Della Valle C, Pulido L, Barrack R (2009) Microbiological, clinical, and surgical features of fungal prosthetic joint infections: a multi-institutional experience. The Journal of bone and joint surgery American 91(Suppl 6):142-149. https://doi.org/ 10.2106/jbjs.I.00574

8. Nace J, Siddiqi A, Talmo CT, Chen AF (2019) Diagnosis and management of fungal periprosthetic joint infections. J Am Acad Orthop Surg 27:e804-e818. https://doi.org/10.5435/ jaaos-d-18-00331

9. Jakobs O, Schoof B, Klatte TO, Schmidl S, Fensky F, Guenther D, Frommelt L, Gehrke T, Gebauer M (2015) Fungal periprosthetic joint infection in total knee arthroplasty: a systematic review. Orthopedic reviews 7:5623. https://doi.org/10.4081/or.2015.5623

10. Schoof B, Jakobs O, Schmidl S, Klatte TO, Frommelt L, Gehrke T, Gebauer M (2015) Fungal periprosthetic joint infection of the hip: a systematic review. Orthopedic reviews 7:5748. https://doi. org/10.4081/or.2015.5748

11. Cobo F, Rodríguez-Granger J, Sampedro A, Aliaga-Martínez L, Navarro-Marí JM (2017) Candida prosthetic joint infection. A review of treatment methods. J Bone Jt Infect 2:114-121. https:// doi.org/10.7150/jbji.17699

12. Akgun D, Perka C, Trampuz A, Renz N (2018) Outcome of hip and knee periprosthetic joint infections caused by pathogens resistant to biofilm-active antibiotics: results from a prospective cohort study. Arch Orthop Trauma Surg 138:635-642. https://doi. org/10.1007/s00402-018-2886-0

13. Insall JN, Thompson FM, Brause BD (1983) Two-stage reimplantation for the salvage of infected total knee arthroplasty. J Bone Joint Surg Am 65:1087-1098

14. Poss R, Thornhill TS, Ewald FC, Thomas WH, Batte NJ, Sledge CB (1984) Factors influencing the incidence and outcome of infection following total joint arthroplasty. Clin Orthop Relat Res:117-126 
15. Karczewski D, Winkler T, Renz N, Trampuz A, Lieb E, Perka C, Muller M (2019) A standardized interdisciplinary algorithm for the treatment of prosthetic joint infections. Bone Joint J 101-B:132139. https://doi.org/10.1302/0301-620X.101B2.BJJ-2018-1056.R1

16. Ueng SW, Lee CY, Hu CC, Hsieh PH, Chang Y (2013) What is the success of treatment of hip and knee candidal periprosthetic joint infection? Clin Orthop Relat Res 471:3002-3009. https:// doi.org/10.1007/s11999-013-3007-6

17. Kuiper JW, van den Bekerom MP, van der Stappen J, Nolte PA, Colen S (2013) 2-stage revision recommended for treatment of fungal hip and knee prosthetic joint infections. Acta Orthop 84:517-523. https://doi.org/10.3109/17453674.2013.859422

18. Gao Z, Li X, Du Y, Peng Y, Wu W, Zhou Y (2018) Success rate of fungal peri-prosthetic joint infection treated by 2 -stage revision and potential risk factors of treatment failure: a retrospective study. Medical science monitor : international medical journal of experimental and clinical research 24:5549-5557. https://doi.org/ 10.12659/msm.909168

19. Cobo F, Rodríguez-Granger J, López EM, Jiménez G, Sampedro A, Aliaga-Martínez L, Navarro-Marí JM (2017) Candida-induced prosthetic joint infection. A literature review including 72 cases and a case report. Infect Dis (Lond) 49:81-94. https://doi.org/10. 1080/23744235.2016.1219456

20. Simonian PT, Brause BD, Wickiewicz TL (1997) Candida infection after total knee arthroplasty. Management without resection or amphotericin B. J Arthroplasty 12:825-829. https://doi.org/10. 1016/s0883-5403(97)90015-2

21. Fusini F, Aprato A, Massè A, Bistolfi A, Girardo M, Artiaco S (2020) Candida periprosthetic infection of the hip: a systematic review of surgical treatments and clinical outcomes. Int Orthop 44:15-22. https://doi.org/10.1007/s00264-019-04369-z

22. Ji B, Zhang X, Xu B, Guo W, Mu W, Cao L (2017) Single-stage revision for chronic fungal periprosthetic joint infection: an average of 5 years of follow-up. J Arthroplasty 32:2523-2530. https:// doi.org/10.1016/j.arth.2017.03.030

23. Vergison L, Schepens A, Liekens K, De Kesel R, Van der Bracht H, Victor J (2020) Periprosthetic joint infection of a total hip arthroplasty with Candida parapsilosis. Int J Surg Case Rep 69:72-75. https://doi.org/10.1016/j.ijscr.2020.03.037

24. Kuo FC, Goswami K, Shohat N, Blevins K, Rondon AJ, Parvizi J (2018) Two-stage exchange arthroplasty is a favorable treatment option upon diagnosis of a fungal periprosthetic joint infection. J Arthroplasty 33:3555-3560. https://doi.org/10.1016/j.arth.2018.07.024

25. Kwong CA, Puloski SK, Hildebrand KA (2017) Fungal periprosthetic joint infection following total elbow arthroplasty: a case report and review of the literature. J Med Case Rep 11:20. https:// doi.org/10.1186/s13256-016-1176-0

26. Evans RP, Nelson CL (1990) Staged reimplantation of a total hip prosthesis after infection with Candida albicans. A report of two cases. J Bone Joint Surg Am 72:1551-1553

27. Açikgöz ZC, Sayli U, Avci S, Doğruel H, Gamberzade S (2002) An extremely uncommon infection: Candida glabrata arthritis after total knee arthroplasty. Scand J Infect Dis 34:394-396. https://doi.org/10.1080/00365540110080232

28. Koutserimpas C, Zervakis SG, Maraki S, Alpantaki K, Ioannidis A, Kofteridis DP, Samonis G (2019) Non-albicans Candida prosthetic joint infections: a systematic review of treatment. World J Clin Cases 7:1430-1443. https://doi.org/10.12998/wjcc.v7.i12.1430

29. Lidder S, Tasleem A, Masterson S, Carrington RW (2013) Candida tropicalis: diagnostic dilemmas for an unusual prosthetic hip infection. J R Army Med Corps 159:123-125. https://doi.org/10. 1136/jramc-2013-000053
30. Younkin S, Evarts CM, Steigbigel RT (1984) Candida parapsilosis infection of a total hip-joint replacement: successful reimplantation after treatment with amphotericin B and 5-fluorocytosine. A case report. J Bone Joint Surg Am 66:142-143

31. Osmon DR, Berbari EF, Berendt AR, Lew D, Zimmerli W, Steckelberg JM, Rao N, Hanssen A, Wilson WR, Infectious Diseases Society of A (2013) Diagnosis and management of prosthetic joint infection: clinical practice guidelines by the Infectious Diseases Society of America. Clin Infect Dis 56:e1-e25. https://doi.org/10. 1093/cid/cis803

32. Zimmerli W, Trampuz A, Ochsner PE (2004) Prosthetic-joint infections. N Engl J Med 351:1645-1654. https://doi.org/10.1056/ NEJMra040181

33. Kienzle A, Walter S, von Roth P, Fuchs M, Winkler T, Muller M (2020) High rates of aseptic loosening after revision total knee arthroplasty for periprosthetic joint infection. JB JS Open Access 5. https://doi.org/10.2106/JBJS.OA.20.00026

34. Kienzle A, Walter S, Palmowski Y, Kirschbaum S, Biedermann L, von Roth P, Perka C, Müller M (2021) Influence of gender on occurrence of aseptic loosening and recurrent PJI after revision total knee arthroplasty. Osteology 1:92-104. https://doi.org/10. 3390/osteology 1020010

35. Diaz-Ledezma C, Higuera CA, Parvizi J (2013) Success after treatment of periprosthetic joint infection: a Delphi-based international multidisciplinary consensus. Clin Orthop Relat Res 471:2374-2382. https://doi.org/10.1007/s11999-013-2866-1

36. Husted H, Holm G, Jacobsen S (2008) Predictors of length of stay and patient satisfaction after hip and knee replacement surgery: fast-track experience in 712 patients. Acta Orthop 79:168-173. https://doi.org/10.1080/17453670710014941

37. Janz V, Wassilew GI, Kribus M, Trampuz A, Perka C (2015) Improved identification of polymicrobial infection in total knee arthroplasty through sonicate fluid cultures. Arch Orthop Trauma Surg 135:1453-1457. https://doi.org/10.1007/s00402-015-2317-4

38. Peel TN, Cheng AC, Buising KL, Choong PF (2012) Microbiological aetiology, epidemiology, and clinical profile of prosthetic joint infections: are current antibiotic prophylaxis guidelines effective? Antimicrob Agents Chemother 56:2386-2391. https://doi.org/10. 1128/AAC.06246-11

39. Kim Y-H, Park J-W, Jang Y-S (2021) Long-term result of a second or third two-stage revision total knee arthroplasty for infected total knee arthroplasty. Arthroplasty 3. https://doi.org/10.1186/ s42836-020-00062-4

40. Oenning S, Moellenbeck B, Gosheger G, Schmidt-Bräkling T, Schwarze J, Ackmann T, Schneider KN, Theil C (2020) Fungal periprosthetic knee joint infection in a patient with metamizoleinduced agranulocytosis. Arthroplast Today 6:726-730. https:// doi.org/10.1016/j.artd.2020.07.040

Institutional review board statement The study was conducted according to the guidelines of the Declaration of Helsinki and approved by the Ethics Committee of Charité University Hospital (EA2/184/17; November 9, 2017).

Publisher's note Springer Nature remains neutral with regard to jurisdictional claims in published maps and institutional affiliations. 